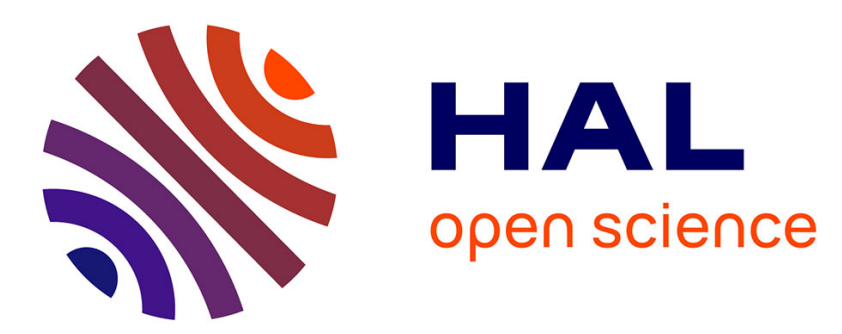

\title{
Les transitions politiques en Mélanésie, éléments de réflexion pour la préparation du " jour d'après" en Nouvelle-Calédonie
}

Carine David

\section{- To cite this version:}

Carine David. Les transitions politiques en Mélanésie, éléments de réflexion pour la préparation du " jour d'après " en Nouvelle-Calédonie. Revue française de droit constitutionnel, 2017. hal-02117083

\section{HAL Id: hal-02117083 \\ https://hal.science/hal-02117083}

Submitted on 2 May 2019

HAL is a multi-disciplinary open access archive for the deposit and dissemination of scientific research documents, whether they are published or not. The documents may come from teaching and research institutions in France or abroad, or from public or private research centers.
L'archive ouverte pluridisciplinaire HAL, est destinée au dépôt et à la diffusion de documents scientifiques de niveau recherche, publiés ou non, émanant des établissements d'enseignement et de recherche français ou étrangers, des laboratoires publics ou privés. 


\title{
Les transitions politiques en Mélanésie, éléments de réflexion pour la préparation du « jour d'après » en Nouvelle-Calédonie
}

\author{
Carine David, MCF HDR, Université de la Nouvelle-Calédonie
}

La Nouvelle-Calédonie est à la croisée des chemins la menant à son destin politique et un nombre croissant de questionnements émergent, au fur et à mesure que l'on approche du terme de la période d'application de l'accord de Nouméa ${ }^{1}$. A l'aube d'un référendum d'autodétermination qui aura a priori lieu en novembre 2018, le temps est venu pour la Nouvelle-Calédonie de procéder à son introspection et de se mettre en capacité de faire des choix. Au-delà de la question clivante posée par le scrutin référendaire, la NouvelleCalédonie doit se doter d'une nouvelle architecture institutionnelle, laquelle devra être déterminée en fonction de la volonté de la société calédonienne ${ }^{2}$ dans la fondation d'un nouveau contrat social ${ }^{3}$, nécessaire dans l'optique de la mise en place d'un statut constitutionnel pérenne, qu'il s'inscrive dans ou en dehors du cadre de la République française.

Dans ce contexte, il parait aujourd'hui nécessaire de déterminer les éléments de méthode permettant de façonner un cadre institutionnel optimal pour répondre au mieux aux spécificités et attentes de la société calédonienne. Dans ce cadre, l'utilisation de référentiels issus du droit comparé apparait pertinente. Néanmoins, la singularité de la situation calédonienne, territoire en voie d'émancipation sur lequel vit une société plurielle, limite considérablement les possibilités de comparaison. Dès lors, la question se pose de l'utilisation de références liées aux processus de transitions constitutionnelles. En d'autres termes, et aux fins de déterminer une méthode optimale, peut-on considérer que la Nouvelle-Calédonie est entrée dans une phase assimilable à une transition constitutionnelle ?

Le caractère transitionnel de la situation politico-institutionnelle de la Nouvelle-Calédonie ne fait aucun doute. Il l'est selon une double dialectique temporelle. D'une part, la NouvelleCalédonie est en phase transitoire depuis 1988 et la signature des accords de MatignonOudinot, voire même depuis plus longtemps si l'on considère le nombre de statuts transitoires qui se sont succédés au cours des années 80 . D'autre part, arrivant au terme de la période

\footnotetext{
${ }^{1}$ L'accord de Nouméa signé le 5 mai 1998 par l'Etat français et les représentants des principaux partis politiques indépendantistes et loyalistes en Nouvelle-Calédonie est un accord politique dont la vocation est de trouver une solution institutionnelle transitoire, dans le cadre d'un processus d'émancipation progressive du territoire. Au terme de cet accord d'une durée d'application de vingt années, un référendum d'autodétermination doit être organisé.

${ }^{2}$ Ainsi, une option en faveur d'un système multiculturaliste ne nécessite pas les mêmes institutions qu'un système intégrationniste par exemple. Voir C. David, « La prise en compte de la diversité culturelle lors des transitions constitutionnelles : analyse à partir du cas des États du Pacifique insulaire », Colloque de l'Association Française des constitutionnalistes, $\quad$ Lyon, 26 juin 2014. http://www.droitconstitutionnel.org/congresLyon/atelierLF.html

${ }^{3}$ S'agissant du lien entre contrat social et constitution, on se réfèrera à J.-P. Massias, « Incidences du processus de pacification sur l'écriture constitutionnelle», in Transitions constitutionnelles et Constitutions transitionnelles - Quelles solutions pour une meilleure gestion des fins de conflit?, éd. Institut universitaire Varenne, coll. Transition \& Justice, 2014, p. 27 à 46.
} 
d'application d'un accord politique conclu en 1998 pour une durée de 20 années, la NouvelleCalédonie se trouve actuellement dans une phase qui se veut terminale de la transition.

Pour autant, peut-on raisonner s'agissant de la Nouvelle-Calédonie en termes de transition constitutionnelle? En d'autres termes, la situation actuelle de la Nouvelle-Calédonie peut-elle être appréhendée comme une situation de nature constitutionnelle? L'idée n'est pas de qualifier la nature de la transition, mais de s'interroger sur la pertinence de l'utilisation de référents constitutionnels. Poser cette question nécessite de s'interroger sur les fonctions particulières d'une constitution dans une société dite «divisée» comme la société calédonienne. Outre ses fonctions classiques déclinées à l'article 16 de la Déclaration des droits de l'homme et du citoyen de 1789, une Constitution y a en effet un rôle fondamental et particulier. "La Constitution peut favoriser le développement d'une identité politique commune en créant les espaces institutionnels pour une prise de décision collégiale entre les membres des différents groupes ethnoculturels. ${ }^{4}$.

Dans un tel cadre, l'acte constitutionnel doit être "un acte d'apaisement collectif et de restauration de la cohésion sociale. Il ne s'agit plus ici de gérer le passé, le présent ou le futur, mais de réconcilier les trois dimensions temporelles. L'enjeu est d'investir le présent afin d'éviter que le passé ne compromette l'avenir $»^{5}$.

Cette appréhension du pacte fondamental d'une société fait écho à une phrase clé de l'accord de Nouméa : «Le passé a été le temps de la colonisation. Le présent est le temps du partage, par le rééquilibrage. L'avenir doit être le temps de l'identité, dans un destin commun »"

Dès lors, il nous semble que la phase qu'est sur le point de traverser la Nouvelle-Calédonie va bien au-delà d'une simple question institutionnelle, au sens de l'évolution statutaire que peut connaitre une collectivité infra-étatique dans un Etat unitaire. En effet, le processus d'émancipation dans lequel est engagée la Nouvelle-Calédonie depuis trois décennies pose inévitablement la question de la définition d'un cadre institutionnel et politique en pratique déconnecté du système constitutionnel français, puisqu'il semble déjà acquis que de nouvelles dérogations constitutionnelles devront être actées ${ }^{7}$ dans l'hypothèse d'une réponse négative au referendum d'autodétermination ${ }^{8}$. A cet égard, il est même envisagé que l'ampleur de ces dérogations justifient de ressusciter l'ancien titre XIII de la Constitution relatif à la communauté. Quoi qu'il en soit, cette étape pose des questions essentielles en termes de choix de société, lesquels auront un impact institutionnel certain dans cette société divisée.

Parmi ces questions, celle de la légitimité de la transition, et partant, du contenu du pacte fondamental se pose avec une particulière acuité. En effet, le choix des procédures utilisées pour réaliser la transition est crucial. Des conférences nationales en passant par les comités

\footnotetext{
${ }^{4}$ S. Choudry, «Bridging comparative politics and comparative constitutional law: Constitutional design in divided societies », in Constitutional Design for Divided Societies - Integration or Accomodation?, Oxford University Press, New York, 2008, p. 6.

5 M. Verdussen, «Préface», in Transitions constitutionnelles et Constitutions transitionnelles - Quelles solutions pour une meilleure gestion des fins de conflit?, op. cit., p. 14.

${ }^{6}$ In Point 4 du Préambule de l'Accord de Nouméa, signé le 5 mai 1998, JONC n 121 du 21 mai 1998, p. 8039.

${ }^{7}$ Voir notamment J. Courtial, F. Mélin-Soucramanien, Réflexions sur l'avenir institutionnel de la NouvelleCalédonie, Rapport au Premier ministre, La Documentation française, Rapports officiels, Paris, 2014, p. 38-39.

${ }^{8}$ Cette question n'aurait pas lieu d'être en cas de réponse positive, synonyme d'accession à la souveraineté.
} 
d'experts ou les «assemblées constituantes », toute une palette de solutions s'offre à la Nouvelle-Calédonie pour déterminer son nouveau contrat social.

Partant de là, la méthode utilisée pour réfléchir aux modalités permettant d'optimiser l'efficacité du processus transitionnel, c'est-à-dire permettant de poser les bases d'un nouveau pacte social, durable et pacifique, s'appuiera nécessairement sur le droit comparé. A titre principal, seront mobilisées des expériences présentant des similitudes avec le contexte calédonien. La détermination des exemples pertinents sera guidée par la nécessité de rechercher des expériences «transposables », en tenant compte de divers éléments tels que notamment les caractéristiques socio-culturelles des sociétés concernées (sociétés traditionnelles / sociétés divisées), la contemporanéité (transitions récentes) ou encore la proximité géographique (sociétés insulaires océaniennes).

Ont dès lors été exclues les expériences océaniennes de transition constitutionnelle lors de leur accession à l'indépendance à partir des années 1960/70. Au contraire, la transition récente à Fidji (2006-2013) ou celle en cours aux Iles Salomon (depuis 2004) seront particulièrement mobilisées.

Il s'agira dans ce cadre de s'intéresser aux acteurs de la transition et plus particulièrement à la place croissante occupée par la société civile (I). Un autre élément important réside dans la gestion du temps dans la transition (II). A cet égard, il semble possible de caractériser une spécificité des processus mélanésiens, pertinente à mobiliser dans le cadre calédonien.

\section{I - Les acteurs de la transition}

«Il ne s'agit pas, dans cette période souvent tendue, de se livrer à un bricolage constitutionnel et de se contenter de quelques aménagements de l'ancien texte ${ }^{9}$. Cette affirmation pose question s'agissant de la Nouvelle-Calédonie alors que le récent rapport de la mission d'écoute et de conseil sur l'avenir institutionnel de la Nouvelle-Calédonie constate le peu de témérité des élus calédoniens pour faire évoluer les institutions locales.

La nécessité de fonder un nouveau pacte social durable et source de paix implique de faire des choix de société qui commanderont alors les choix institutionnels. Dès lors, il importe de connaître la volonté de la population, de prendre le pouls de la société. Ce passage obligé est d'autant plus important dans une société clivée comme la société calédonienne afin de tendre vers la satisfaction du plus grand nombre d'une part, et de favoriser la construction nationale d'autre part.

Comme dans l'ensemble des processus de transition démocratique, il s'agit donc ici de déterminer les modalités de discussion/négociation permettant de définir une telle ingénierie institutionnelle et partant, de poser la question de la place de la société civile dans le processus, non seulement s'agissant de sa légitimité mais également du point de vue de son appropriation par la société dont le statut entend régir le fonctionnement. Dans cette perspective, il convient de s'interroger sur les modalités et l'efficacité de cette participation (A) et d'en tirer des enseignements pour déterminer l'organisation d'un processus de transition adapté aux réalités calédoniennes (B).

\footnotetext{
${ }^{9}$ X. Philippe, «Tours et contours des transitions constitutionnelle - Essai de typologie des transitions », in Transitions constitutionnelles et Constitutions transitionnelles - Quelles solutions pour une meilleure gestion des fins de conflit ?, op. cit., p. 19.
} 


\section{A - La place évolutive de la société civile dans les processus transitionnels}

Globalement, les processus transitionnels au moment de la décolonisation, que ce soit en Afrique $^{10}$ ou en Océanie ${ }^{11}$, caractérisés par la notion de «mimétisme constitutionnel ${ }^{12}$, n'ont laissé que peu, voire aucune place à la société civile. En effet, les procédures mobilisées ont consisté en une gestion de la transition par l'Etat colonial sur le départ et le contenu des constitutions a été calqué sur celui des systèmes occidentaux, de manière totalement déconnectée des spécificités socioculturelles des populations concernées. Ainsi, pratiquement tous les Etats insulaires du Pacifique ont choisi au moment de l'indépendance une forme constitutionnelle proche de celle existant pendant la période coloniale, elle-même étant un décalque aménagé des systèmes constitutionnels de l'Etat colonial ${ }^{13}$.

$\mathrm{Au}$ contraire, les processus plus récents de transition en Océanie comme en Afrique démontrent une évolution importante dans la prise en considération des souhaits exprimés par la société civile. En effet, que l'on se réfère au processus transitionnel en Afrique du Sud, aux procédures mises en place au Maghreb suite au «printemps arabe » ou aux réformes récentes (Fidji) ou en cours (Iles Salomon) en Océanie, il apparait clairement que la participation directe de la société civile à la transition est jugée incontournable par les pouvoirs publics.

Toutefois, ces approches inclusives et participatives sont aujourd'hui peu mobilisées dans le cadre du processus en cours en Nouvelle-Calédonie. Une telle approche s'inscrirait pourtant logiquement dans l'évolution des modalités de détermination du contenu des statuts successifs de la Nouvelle-Calédonie qui peut être caractérisé en distinguant deux grandes périodes.

Une première période (1853-1980) correspond à une époque où l'Etat détermine unilatéralement les statuts ; la Nouvelle-Calédonie a alors un statut de colonie, puis de collectivité ultramarine de droit commun. On raisonne alors classiquement par rapport à la détermination du statut d'une collectivité territoriale dans un Etat unitaire : l'Etat détermine le degré d'autonomie dont bénéficie la collectivité et l'architecture institutionnelle qui l'accompagne.

Une deuxième période, postérieure à 1980, voit l'évolution vers une concertation tripartite (Etat - représentants politiques indépendantistes - représentants politiques loyalistes) dans un cadre d'émancipation et de revendications sécessionnistes. Depuis les années 80, l'Etat a dû négocier pour tenter de satisfaire les revendications des représentants indépendantistes sans céder à la demande de sécession.

10 C. Danelciuc-Colodrovschi, «L'incidence des influences constitutionnelles externes sur l'écriture et l'adoption des constitutions postconflictuelles», in Transitions constitutionnelles et Constitutions transitionnelles - Quelles solutions pour une meilleure gestion des fins de conflit?, p. 113.

${ }^{11}$ C. David, « La prise en compte de la diversité culturelle lors des transitions constitutionnelles : analyse à partir du cas des États du Pacifique insulaire », Colloque de l'AFDC, Lyon, 26 juin 2014. Téléchargeable sur le site de l'AFDC.

12 J. du Bois de GAUDUSSON, «Le mimétisme post colonial, et après ? », Pouvoirs 2009/2, pp. 45-55. V. aussi, Y. MENY (dir.), Les politiques du mimétisme juridique. La greffe et le rejet, Paris, L’Harmattan, 1993.

${ }^{13}$ Ainsi, les anciennes colonies britanniques ou associées à l'Australie ou à la Nouvelle-Zélande ont mis en place des régimes parlementaires, de type Westminster alors que ceux associés aux Etats-Unis ont choisi un régime présidentiel. 
Un aperçu historique rapide permet de percevoir l'évolution des modalités de la négociation pendant cette période. Les discussions de Nainville les Roches en 1983 marquent l'ébauche du processus de transaction tripartite. En 1988, les tractations pour la conclusion de l'accord de Matignon pérennisent cette solution triangulaire négociée, le contenu de l'accord étant au surplus validé par référendum national. Enfin, le dernier stade d'évolution est marqué par la signature de l'accord de Nouméa en 1998, toujours négocié par des délégations représentant les deux tendances politiques locales et l'Etat, ce dernier optant pour une posture de facilitateur et d'arbitre. L'accord sera ensuite approuvé par référendum local.

On constate donc un effacement progressif du rôle de l'Etat corrélativement à une augmentation graduelle mais timide de l'engagement de la société civile, ce qui correspond à la tendance observée dans les processus transitionnels depuis une vingtaine d'années, où l'on est passé de modalités privilégiant des sessions closes entre élites politiques à des processus de construction de la Constitution renforçant l'engagement de la société civile.

A cet égard, les processus utilisés sont devenus cruciaux dans la réussite ou l'échec des transitions démocratiques, particulièrement dans les sociétés divisées ethniquement puisque dans l'idéal, la construction de la Constitution est un effort minutieux pour une réforme socio-politique et la résolution des conflits.

Les modalités de la transition, la manière dont les acteurs politiques sont organisés et les différentes institutions qui émergent durant le processus transitionnel sont fondamentaux, s'agissant de la viabilité à long terme de l'organisation démocratique d'un Etat ou territoire. Le droit international et le droit comparé ont progressivement fait émerger des «bonnes pratiques » en la matière.

Un premier principe réside dans le caractère inclusif des négociations ${ }^{14}$ : toutes les voix $-\mathrm{y}$ compris les opposants politiques, les organisations de la société civile et les minorités - sont écoutées et mises en valeur dans le processus.

Il en découle un deuxième principe qui est celui de la participation $^{15}$ : la population est formée au processus et doit pouvoir effectivement s'exprimer. La participation doit s'entendre comme bien plus que d'ouvrir des espaces pour le dialogue. Cela implique d'analyser les buts du processus de participation et conférer les compétences qui donne la capacité aux citoyens de participer effectivement et avec sens au processus.

La participation a plusieurs fonctions ${ }^{16}$. Elle a tout d'abord une vocation unificatrice par son inclusivité qui permet que le processus se déroule dans la confiance entre les personnes appartenant aux différentes communautés. Elle améliore par ailleurs la légitimité du statut ou de la Constitution : le processus et le document final sont légitimes aux yeux du public, réduisant par là le risque d'instabilité pendant le processus transitionnel. Elle permet enfin, en

\footnotetext{
${ }^{14}$ Voir notamment M. Besse, «L’écriture multilatérale des constitutions, facteur de réussite des transitions démocratiques », Nouveaux Cahiers du Conseil constitutionnel, $\mathrm{n}^{\circ}$ 45, 2014 ; K. Samuels, « Post-conflict peacebuilding and constitution-making », Chicago Journal of International Law, n², 2006, p. 2-20 ; Annual review of constitution-building processes 2014, Institue for democracy and electoral assistance (IDEA), 2015.

${ }^{15}$ T. Ginsburg, «Introduction », in Comparative Constitution Design, Cambridge University Press, 2012, p. 112 ; C. Saunders, «Constitution making in the $21^{\text {st }}$ century », International review of law, 2014.

${ }^{16}$ R. Kant, «Constitution making and transition to democracy in Fiji », presentation lors de la Conférence de la Pacific Islands Political Studies Association (PIPSA) Conference, Université de la Polyénsie française, Papeete, 2014.
} 
initiant une citoyenneté active et informée, une appropriation du processus transitionnel par la population, élément déterminant s'agissant de la stabilité du régime à long terme.

La transparence constitue par ailleurs un troisième principe : le public doit être informé de ce qu'il se passe à toutes les étapes du processus.

Enfin, pour être efficace, le processus doit reposer sur le consensus et être en conséquence basé sur la discussion, la négociation, la persuasion.

Dans ce cadre, et comme le souligne M. Besse ${ }^{17}$, l'analyse des processus transitionnels soulève l'inefficacité des processus constituants non inclusifs et des processus constituants dits «antagonistes », c'est-à-dire dans lesquels on constate une incapacité des acteurs à résoudre leurs différences pour faire aboutir le processus sur la base d'une décision prise en commun. Les deux principaux écueils sont le risque de transition constitutionnelle avortée ou celui d'une victoire d'un camp sur l'autre.

Dans cette optique, il convient de s'attacher à déterminer les différentes modalités d'organisation d'un processus transitionnel associant la société civile calédonienne.

B - L'organisation de la participation citoyenne dans les processus constituants

Outre les options de structuration de cette participation sociétale, il y a lieu de s'interroger sur la pertinence et l'effectivité de cette participation.

1. La pertinence et l'effectivité de la participation citoyenne

Il existe aujourd'hui un consensus quant au fait que plus les citoyens seront impliqués dans la détermination du contenu de la nouvelle constitution, plus légitime sera considérée la transition. ${ }^{18}$ Dès lors, il convient de s'intéresser à l'organisation de la participation citoyenne pour en optimiser l'efficacité.

Un premier élément récurrent réside dans la nécessité de sensibilisation et de formation de la population aux enjeux de la réforme. Certains auteurs évoquent une «alphabétisation constitutionnelle $»^{19}$. Si l'implication de la société civile est réellement voulue comme effective, il convient préalablement d'organiser une campagne d'éducation civique permettant à la population de participer le plus pertinemment possible. Durant cette phase, il y a lieu de mobiliser des outils adaptés aux populations auxquelles on souhaite s'adresser et de varier les supports. Seront ainsi mobilisés les forums décentralisés, la presse, la radio, la télévision, internet, la diffusion de brochures éducatives.

Cette première phase passée, il existe différentes modalités d'organisation du processus participatif.

Il faut tout d'abord opéré un choix quant à l'existence ou non d'un pré-projet de constitution utilisé comme base de discussion, lequel sera modifié en fonction des objections formulées dans le cadre de la participation.

A cet égard, l'exemple salomonais est particulièrement intéressant. Les citoyens ont été sollicités sur la base d'un premier pré-projet de Constitution rédigé en 2004. Les versions

\footnotetext{
${ }^{17}$ M. Besse, op. cit.

${ }^{18}$ M. Besse, op. cit.

19 R. Déchaux, «La légitimation des transitions constitutionnelles », », in Transitions constitutionnelles et Constitutions transitionnelles - Quelles solutions pour une meilleure gestion des fins de conflit ?, p. 195.
} 
successives du projet ont été ensuite modifiées en tenant compte des remarques/réserves émises lors des réunions publiques et synthétisées dans des rapports élaborés par chaque comité provincial, selon la méthode de l'entonnoir, seuls les points restant encore en discussion étant envisagés à chaque stade du processus.

Une autre possibilité réside dans l'absence de projet support. Dans ce cas, la participation prend généralement la forme de contributions, la population pouvant émettre des propositions sur des sujets divers et variés. Les difficultés liées à la rationalisation d'un tel processus sont néanmoins évidentes tant le nombre de propositions émises peut être important et leur contenu contradictoire. Par ailleurs, le risque est alors important pour que les propositions formulées ne portent pas à proprement parler sur des éléments d'une Constitution mais sur des revendications sociales plus générales. ${ }^{20}$

Cette façon de procéder, si elle permet une grande liberté des citoyens, pose la question de l'effectivité de la prise en compte des propositions citoyennes. Elle laisse en effet une grande marge de manœuvre aux structures institutionnelles mises en place pour opérer une sélection par un tri des propositions sur des critères non nécessairement transparents. Dans un tel cadre, la participation semble plus un moyen de permettre un «état des lieux des revendications de la population à un moment clé de son histoire ${ }^{21}$. On rejoint alors R. Déchaux, lorsqu'il estime qu'il faut alors considérer que "la démocratie est surtout un esprit qui n'est ni majoritaire, ni participatif, et qui repose sur la tolérance plus que sur celle de la représentation mathématique des intérêts particuliers $»^{22}$.

\section{La structuration de la participation citoyenne}

Si l'intérêt de la participation citoyenne semble acquis, le choix de la structure sera l'occasion de déterminer la place concrètement accordée à la société civile, et par là celle de la légitimité du processus. Ainsi, la composition de la structure et les modalités de désignation de ses membres sont des éléments cruciaux pour la réussite de la réforme. Pour autant, certains auteurs $^{23}$, tel $\mathrm{Y}$. Ghai ${ }^{24}$, soulèvent le risque de contre productivité de la participation dans le cadre d'une société divisée.

\section{a) Les options de structuration}

Quel que soit le degré d'implication de la société civile, la forme que prend la participation citoyenne et le moment auquel celle-ci a lieu, les expériences récentes de transition démontrent la nécessité d'un cercle unique, central, au sein duquel l'ensemble des acteurs sont réunis pour prendre les principales décisions. ${ }^{25}$ La participation des citoyens doit en pratique nécessairement être rationnalisée, faute de quoi la voix du peuple ne sera pas audible. On comprend dès lors que la composition d'une telle structure revêt une importance capitale en termes de légitimité.

\footnotetext{
${ }^{20}$ R. Déchaux, op. cit., p. 190.

${ }^{21}$ R. Déchaux, op. cit., p. 195.

${ }^{22}$ R. Déchaux, op. cit. p. 194.

${ }^{23}$ Voir notamment : Making Constitutions in Deeply Divided Societies, Hanna Lerner, Cambridge University Press, 2011.

${ }^{24}$ J. Cottrell \& Y. Ghai, «Constitutional engineering and impact: the case of Fiji » in Arjomand (dir.) Constitutionalism and political reconstruction. Brill. 2007, p. 159-192; Making Constitutions in Deeply Divided Societies, op. cit.

${ }^{25}$ Constitutional Development with civil society - Case studies from Southern and East Africa, S. T. Panfil (dir.), http://www.constitutionnet.org/vl/item/constitutional-development-civil-society-case-studies-southernand-east-africa
} 
De manière surprenante, le droit comparé nous enseigne que l'élection n'est pas nécessairement le mode le plus pertinent car le risque est important qu'elle ne garantisse pas le multilatéralisme ${ }^{26}$.

De façon générale, une solution privilégiée consiste en un organe composé de membres choisis pour leur représentativité dont la crédibilité repose alors non pas sur la volonté populaire via le vote mais sur l'accord des membres reconnus de l'opposition et des différents groupes composant la société civile de l'intégrer.

A cet égard, la convention pour la démocratie en Afrique du Sud constitue un exemple intéressant. Pour le Président de Klerk, la négociation de la nouvelle Constitution relevait de la responsabilité des représentants de tous les partis politiques bénéficiant d'un soutien populaire et s'étant engagés dans la recherche d'une solution pacifique et négociée ${ }^{27}$. Alors qu'il apparaissait évident que l'élection d'une assemblée constituante aurait donné une victoire écrasante à l'ANC et par conséquent une absence de multilatéralisme qui aurait entrainé un risque fort d'un rejet de la transition, fut créée la COSEDA (Convention for a Democratic South Africa) composée de représentants non élus réunissant le Gouvernement national, les administrations des 10 bantoustans ${ }^{28}$ et 7 partis politiques, dont le parti national, l'ANC et l'Inkhata (parti zoulou). Preuve de la crédibilité de cette structure, d'autres partis d'extrême droite rejoignirent la convention par la suite après avoir initialement refusé de s'associer aux négociations.

La mise en place de telles structures permettent la réunion de l'ensemble des acteurs de la transition.

Notons par ailleurs, qu'il apparait qu'une nature politique des organes de négociation est préférable en ce que l'absence de compétences normatives facilite la prise de décision unanime ou par consensus. Les décisions ayant une portée purement politique, elles seront converties ultérieurement en normes par une autre institution ${ }^{29}$.

\section{b) La question de la participation dans les sociétés divisées}

Néanmoins, selon Y. Ghai ${ }^{30}$, dans les sociétés divisées, la participation présenterait un fort risque de contre productivité. En effet, il y existerait un risque accru que chaque groupe recherche ses intérêts propres et ne soit pas préparé à travailler avec les autres groupes pour discuter sérieusement du futur et résoudre les conflits. Par ailleurs, dans un tel contexte, les discussions auraient tendance à être étroites et à tourner autour de points particuliers, ce qui ralentirait d'autant plus le processus.

En conséquence, il existerait une tendance dans les sociétés divisées à ce que les décisions soient prises par un petit groupe d'élites influentes, ayant pour conséquence un processus souvent non participatif et non transparent. C'est d'ailleurs vers une telle solution que les élus

\footnotetext{
${ }^{26}$ Les expériences égyptienne (voir notamment M. Besse, op. cit.) ou en Afrique du Sud.

27 C. Salcedo, La transition démocratique sud-africaine: essai sur l'émergence d'un droit public de la reconstruction de l'Etat, Fondation Varenne, Collection de thèses, 2011, p. 39.

${ }^{28}$ Gouvernements traditionnels dans les régions réservées aux populations noires pendant l'apartheid.

${ }^{29}$ Contre-exemple : Bénin où la conférence nationale souveraine béninoise, composée des forces vives de la Nation non élues, était à la fois politique et constituante. Voir M. Besse, op. cit.

${ }^{30}$ J. Cotrell \& Y. Ghai, op. cit.
} 
calédoniens semblent une nouvelle fois s'orienter. En effet, les discussions ont actuellement lieu dans le cadre du «comité des signataires », composé des signataires de l'accord de Nouméa, auxquels ont été ajoutés certaines personnalités politiques. Assisté d'experts personnalités qualifiées désignées par l'Etat français - cette structure ne bénéficie d'aucune légitimité populaire et élude la question de la structuration d'une participation citoyenne, chaque parti politique se contentant d'organiser des forums d'information sur leur vision de l'avenir institutionnel.

Pourtant, tout semble laisser penser que la société calédonienne est prête pour un processus transitionnel l'associant plus étroitement. En effet, on peut souligner que l'expérience du consensus dans la vie politique calédonienne permet de penser que les populations sont en capacité d'assumer un tel processus. L'expérience acquise par le biais des négociations statutaires successives depuis le début des années 80, la pratique depuis 1999 de la prise de décision exécutive dans le cadre d'un gouvernement désigné à la représentation proportionnelle par le Congrès de la Nouvelle-Calédonie, ainsi que les discussions annuelles dans le cadre du comité des signataires de l'accord de Nouméa pour régler les difficultés soulevées par la mise en œuvre de l'accord politique sont autant d'éléments rythmant la vie politique locale. Ils ont permis à la société calédonienne d'intégrer la nécessité continue de la pratique du consensus et d'acquérir une certaine maturité en la matière. Tous ces éléments semblent propices à la mise en place d'une forme de participation dans le processus transitionnel calédonien.

Par ailleurs, il existe des exemples récents de processus participatifs réussis dans des sociétés divisées. On pense bien sûr à l'Afrique du Sud mais également au processus en cours aux Iles Salomon.

La société salomonaise est une société divisée sur une base linguistique. En effet, les salomonais vivent traditionnellement en petits groupes autonomes. Leur loyauté ne va pas audelà des groupes de personnes parlant la même langue ${ }^{31}$ ou originaires de la même île et il n'existe aucune structure sociale les englobant, pour permettre l'unité. ${ }^{32}$

Suite à des conflits ethniques meurtriers s'étant déroulés à la fin des années 90, le gouvernement des Iles Salomon a décidé de réformer sa Constitution, datant de l'accession à l'indépendance en 1978.

Dans cette optique, un congrès constitutionnel (Constitutional Congress) a été mandaté comme organe central pour travailler sur la réforme. La composition de cette structure a été pensée comme inclusive et participative en incluant des représentants des provinces, nommés par les exécutifs provinciaux et de représentants nationaux pour exprimer la volonté des différents groupes de la société civile (jeunes, anciens, femmes...). Ce comité a été associé à une autre structure : l'Eminent Persons Advisory Council (EPAC) composée de personnalités politiques expérimentées et de représentants de toutes les communautés des Iles Salomon. Ces deux structures ont travaillé de concert tout au long du processus, en interaction directe et continue avec la population salomonaise. Des échanges réguliers avec la population, via des consultations au niveau provincial, ont en effet été organisés à chaque stade de la procédure.

\footnotetext{
${ }^{31}$ Il existe plus de 70 groupes linguistiques aux Iles Salomon.

${ }^{32}$ H. Laracy (dir.), Ples Blong Lumi : Salomon Islands, the past four thousand years, Institute of Pacific Studies, University of South Pacific, Suva, 1989.
} 
Le processus est en voie d'achèvement, l'adoption de la Constitution étant prévue pour le courant de l'année 2017.

L'expérience salomonaise constitue un excellent exemple de structuration réussie de la participation citoyenne dans le cadre d'un processus transitionnel dans une société divisée. La version finale du projet de Constitution a en effet reçu l'assentiment de l'ensemble des neufs comités provinciaux, constitué dans chaque province, ainsi que de l'ensemble des diasporas installées dans divers Etats du Pacifique, consultées dans le cadre d'un programme très étendu d'information et de participation sur la réforme constitutionnelle organisé tout au long de l'année 2015 sur la dernière version du projet de Constitution. ${ }^{33}$

Au-delà de la place effectivement accordée à la société civile qui, on le voit, se pose de façon spécifique dans les sociétés divisées, il apparait que les processus transitionnels mélanésiens peuvent être caractérisés par une appréhension du temps différente, tout en étant en totale adéquation avec la culture océanienne dans le cadre de laquelle le temps fait l'objet d'une perception toute particulière, notamment s'agissant de la prise de décision. En effet, la recherche $\mathrm{du}$ consensus est un élément constant dans les sociétés traditionnelles mélanésiennes. En conséquence, on peut certainement caractériser les processus mélanésiens comme étant inscrits dans des temps longs.

\section{II - Le temps dans le processus transitionnel : la « Melanesian way » de la transition}

Au-delà de la question des participants au processus transitionnel, un élément omniprésent réside dans le temps ${ }^{34}$. Dans ce cadre, la notion de temps peut être appréhendée sous deux angles, lesquels s'avèrent inextricablement liés : celui de la durée du processus (A), mais également celui du moment auquel doit s'opérer le processus (B). De ces deux points de vue, les expériences mélanésiennes apparaissent particulièrement pertinentes au regard de la situation calédonienne. Elles semblent caractériser une «Melanesian way » de la transition dans le cadre de laquelle l'appréhension océanienne du temps joue un rôle privilégié dans le sens où les processus sont inscrits dans la durée. A cet égard, il apparait que l'on assiste actuellement à un mouvement tardif d'émancipation institutionnelle des Etats insulaires mélanésiens.

A - La durée des processus transitionnels mélanésiens

En Mélanésie, on peut souligner l'écoulement d'un temps certain entre l'évènement déclencheur du processus, le déclenchement effectif de la procédure et la conclusion du processus.

Ainsi, en Nouvelle-Calédonie, les évènements des années 80 ont conduit à la mise en place d'un statut transitoire pour dix ans avec la signature des accords de Matignon-Oudinot en novembre 1988. Jugeant que la population calédonienne n'était pas prête à être confrontée à un référendum d'autodétermination dès 1998, les responsables politiques locaux ont alors fait

\footnotetext{
${ }^{33}$ Voir le site officiel de la réforme : http://www.sicr.gov.sb/

${ }^{34}$ D. Rousseau, «Le temps de la Constitution dans les transitions politiques », in Transitions constitutionnelles et Constitutions transitionnelles - Quelles solutions pour une meilleure gestion des fins de conflit ?, p. 225.
} 
le choix d'inscrire encore un peu plus ce processus dans la durée avec la conclusion de l'accord de Nouméa le 5 mai 1998, renvoyant à 20 ans la tenue d'un référendum d'autodétermination.

Aux Iles Salomon, les conflits meurtriers du fait de tensions ethniques entre 1998 et 2000 liées à des rivalités foncières entre les populations de Guadalcanal et des populations originaires d'autres régions (notamment Malaïta) ont constitué le déclencheur d'un processus de révision constitutionnelle en ce qu'ils ont permis d'exprimer l'inadéquation du système mis en place par la Constitution d'indépendance de 1978 mettant en place un Etat unitaire sous la forme d'une monarchie parlementaire sur le modèle britannique dans une société très hétérogène, notamment d'un point de vue linguistique.

Suite à la signature du Townsville Peace Agreement Act 2000, le Gouvernement salomonais a pris le temps de la rédaction d'une première version d'un projet de constitution qui a été diffusé en $2004^{35}$, avant d'établir les modalités du processus dans un livre blanc en 2005. Les structures de discussion, au nombre de deux, ont été mises en place en 2007.

En 2008, un premier travail d'identification des «communautés politiques » a commencé, consistant, dans chaque province, à identifier des personnes ressources à consulter. Cette étape a été suivie de la mise en place de comités thématiques ( $\mathrm{DH}$, finances, justice, institutions, partage des pouvoirs...). Néanmoins, les membres des différents comités, estimant qu'une vision d'ensemble était nécessaire pour appréhender au mieux le processus, ont souhaité assister à l'ensemble des discussions. Dès lors, l'ensemble des réunions ont été tenues en formation plénière.

En 2009, une deuxième version du projet de Constitution a été arrêté par le congrès constitutionnel et l'EPAC. Ce projet a ensuite été soumis aux comités provinciaux en 2010, dont les rapports ont alors permis de faire remonter les appréciations sur le projet, via les équipes provinciales du congrès constitutionnel, aboutissant à une nouvelle version du projet de constitution en 2011. L'année suivante, des conventions provinciales ont été organisées afin de consulter le public sur le projet. A cette occasion, les communautés salomonaises à l'étranger (Fidji, Vanuatu) ont également été consultées.

En 2013, une nouvelle version du projet de constitution, intégrant les remontées des conventions provinciales a été validée par le congrès constitutionnel et l'EPAC. Le projet a alors été audité par des experts extérieurs, parmi lesquels le constitutionnaliste Y. Ghai, artisan de plusieurs constitutions, notamment dans le Pacifique insulaire.

En 2014, une nouvelle réunion plénière du congrès constitutionnel et de l'EPAC a été l'occasion de valider une nouvelle version du projet après sept semaines de travail sur les retours des experts et en leur présence. Cette version du projet a alors été soumise aux équipes provinciales du congrès constitutionnel et un large programme d'information et de participation du public ( «Public Awareness Program ») a eu lieu pendant toute l'année 2015.

Une dernière session plénière des deux organes dédiés à la réforme a permis en 2016 d'arrêter la rédaction finale du projet de Constitution, avant transmission au Premier ministre, les modalités d'adoption définitive de la Constitution restant à définir.

\footnotetext{
${ }^{35}$ J. Corrin, «Breaking the mould : constitutional review in Salomon Islands », Revue juridique polynésienne, $\mathrm{n}^{\circ} 13,2007$, p. 143 et $\mathrm{s}$.
} 
Initialement prévu d'être mené à son terme en 2015, le processus est donc toujours en cours aujourd'hui et devrait se clôturer par l'adoption de la nouvelle Constitution de la République fédérale des Iles Salomon en 2017, après treize années de travail, sept versions successives et d'intenses campagnes d'information et de participation tout au long du processus.

A Fidji, le coup d'Etat de décembre 2006 a été le point de départ de la remise en cause de la Constitution de 1997 qui n'a jamais vraiment été mise en œuvre. Le coup d'Etat, justifié par son auteur par la nécessité de passer outre les divisions ethniques au sein de la société fidjienne, a toujours été considéré comme un moyen de rebattre les cartes du jeu politique pour aboutir à la réforme du système constitutionnel, jugé trop multiculturaliste. Dès lors, ne cédant pas à la pression de la communauté internationale lui enjoignant un retour rapide à la démocratie par l'organisation d'élections législatives, l'auteur du coup d'Etat, F. Bainimarama a annoncé en 2009 un calendrier beaucoup plus étalé dans le temps. Arguant de la nécessité d'un certain nombre de réformes sur des problématiques clivantes, préalablement à toute réforme constitutionnelle, sous peine de la conduire à l'échec, le Premier ministre autoproclamé définit un calendrier reportant la date de retour à la démocratie à 2014 .

Rédigée après un processus inclusif et participatif, néanmoins non exempt d'incidents, la Constitution de la République des Iles Fidji de 2013 met en place un régime constitutionnel expurgé de toute référence clivante sur une base ethnique, en rupture avec les constitutions précédentes. Conformément au calendrier annoncé cinq ans plus tôt, des élections législatives ont été organisées en septembre 2014.

On le voit, les processus transitionnels mélanésiens s'échelonnent sur des périodes relativement longues, exprimant un trait caractéristique de la prise de décision dans le Pacifique insulaire: la recherche systématique du consensus, laquelle implique des discussions longues. Le palabre constitue un élément fondamental de la prise de décision. Il implique la nécessité de discuter jusqu'à trouver un terrain d'entente, peu importe le temps que nécessite la prise de décision consensuelle. Dans ce cadre culturel particulier, le facteur temps n'est pas un critère essentiel dans la prise de décision. Il est considéré que pour être acceptée et donc appliquée car appropriée et ressentie comme légitime, la décision doit recueillir l'assentiment du plus grand nombre, même si atteindre le consensus signifie prendre le temps.

Cette appréhension du temps peut être également décelée dans le délai mis par les Etats insulaires pour se démarquer des institutions et du régime politique hérités de la colonisation, les Etats mélanésiens semblant connaitre actuellement un processus d'émancipation institutionnelle, plusieurs décennies après l'émancipation politique. A cet égard, la NouvelleCalédonie se situe à rebours de ce processus.

B - Des processus transitionnels récents marquant l'émancipation tardive des Etats insulaires mélanésiens

Conflit de type guerre civile ou coup d'Etat sont les deux éléments déclencheurs des transitions politiques que l'on peut trouver dans l'histoire récente océanienne.

En Nouvelle-Calédonie, «les évènements » des années 80 opposant les indépendantistes kanak aux partisans du maintien dans la France constitue une expression pudique pour ne pas exprimer le terme de guerre civile. Le même type de conflits internes est à l'origine du 
processus actuellement en cours aux Iles Salomon. A Fidji, la division de la société exprimée au plus haut niveau par des responsables politiques dans l'incapacité de passer outre leurs oppositions pour faire fonctionner les institutions a été à l'origine d'un coup d'Etat orchestré par le chef des armées, excédé de la paralysie du régime depuis plusieurs années.

A y regarder de plus près, les processus transitionnels récents, en cours ou en prémisse en Mélanésie semblent marquer une émancipation tardive de ces petits Etats insulaires vis-à-vis de l'ancienne puissance coloniale, comme si la décolonisation institutionnelle ne s'opérait que plusieurs décennies après l'émancipation politique.

En effet, si l'on exclut la Papouasie-Nouvelle-Guinée qui est justement le seul Etat insulaire de Mélanésie à avoir mis en place un cadre constitutionnel déconnecté de celui de l'Etat colonial sur le départ, les trois autres Etats de la Mélanésie, à savoir Fidji, les Iles Salomon et le Vanuatu, se sont dotés d'une Constitution reprenant les caractéristiques du système Westminster d'une part et perpétuant les clivages sociétaux créés par la puissance coloniale d'autre part.

Ainsi, jusqu'à la Constitution de 2013, la République des Iles Fidji ne s'est jamais réellement émancipée de l'institutionnalisation des clivages socio-culturels entretenus par les Britanniques pendant la période coloniale entre les autochtones mélanésiens d'un côté et les indo-fidjiens, issus de l'immigration du travail de l'autre. La Constitution d'indépendance de 1970, rédigée à Londres, avait été conçue pour entretenir cette fragmentation socio-culturelle par la mise en place d'outils institutionnels clivant. La Constitution «scélérate » de 1990, rédigée après deux coups d'Etats perpétrés en 1987 au nom de la sauvegarde des intérêts de la population autochtone, avait d'ailleurs accentué cette division en mettant en place un cadre constitutionnel favorisant les autochtones mélanésiens et excluant les indo-fidjiens de tous les postes politiques à responsabilité. Quant à la Constitution de 1997 dont l'application sera ponctuée par deux coups d'Etat en 2000 et 2006, elle ne permettra pas de sortir de la dichotomie sociétale née de la période coloniale. Le cadre constitutionnel qu'elle mettait en place, pourtant plus égalitaire, a immédiatement montré ses limites tant les concessions acceptées par les représentants politiques mélanésiens reposaient sur la certitude - non avérée par la suite - que la supériorité numérique de la population autochtone permettrait à elle-seule d'assurer la suprématie du peuple originaire. Néanmoins, la volonté populaire d'aller au-delà du clivage socio-culturel pour s'engager dans un processus de construction nationale s'est heurtée à l'intransigeance de responsables politiques incapables de passer outre une vision clivée de la société fidjienne sur une base ethnoculturelle. C'est la Constitution de 2013 qui opère le nécessaire dépassement de cette vision binaire dans le cadre constitutionnel et qui pourrait permettre un véritable élan de construction nationale, décomplexée de son passé colonial.

Le cas des Iles Salomon est différent mais reflète cette même difficulté de l'Etat de s'émanciper du cadre imposé par l'ancienne puissance coloniale. La Constitution de 1978, toujours en vigueur aujourd'hui, est une caricature du «mimétisme constitutionnel » ${ }^{36}$ opéré lors de l'accession de nombre de possessions coloniales dans les années 60 et 70 en Afrique comme en Océanie. Monarchie constitutionnelle dont le chef de l'Etat reste la reine Elizabeth II, les Iles Salomon sont un Etat unitaire doté d'un régime parlementaire proche du système Westminster, jusque dans le choix du mode de scrutin aux élections législatives. En effet, le

${ }^{36}$ J. du Bois de GAUDUSSON, « Le mimétisme post colonial, et après ? », op. cit. 
système du «First Past The Post» mis en place dans le pays s'avère totalement inadapté dans le cadre d'une population très hétérogène et traditionnelle, dans un système politique totalement dépourvu de structuration et donc de partis politiques. La conséquence en est que le mode de scrutin produit des effets totalement inverses à ceux que l'on connait dans les démocraties occidentales, induisant une instabilité institutionnelle chronique et rendant difficile le gouvernement du pays.

Au contraire, le projet de Constitution, revendiqué par le gouvernement Salomonais comme un texte «home grown » et qui devrait être adopté dans le courant de l'année 2017 prend le total contrepied du système constitutionnel existant. Il est ainsi probable que les Iles Salomon, s'émancipant de la Reine Elizabeth II, mue en République fédérale, sous la forme d'un régime parlementaire et dont le mode de scrutin privilégié sera le vote alternatif, tout en optant pour une meilleure prise en compte des structures coutumières chères aux populations traditionnelles.

Enfin, et plus rapidement, le Vanuatu semble à son tour se préparer à une introspection et à tirer les conséquences des dysfonctionnements générés par la Constitution d'indépendance de 1980, toujours en vigueur. On peut très certainement considérer que la situation vanuataise constitue la synthèse des difficultés rencontrées par ses deux voisins. Outre le clivage de la société entre francophones et anglophones, né du statut original de condominium francobritannique sous la double responsabilité de la France et de la Grande-Bretagne pendant la période coloniale, le décalque du système institutionnel westminsterien inadapté aux spécificités socio-culturelles, linguistiques et géographiques du pays a, là encore, pour conséquence une instabilité gouvernementale chronique.

Le processus de révision constitutionnelle n'a toutefois été que récemment envisagé, le Premier ministre élu en février 2016 proposant d'initier de larges consultations afin de procéder à la réforme du cadre constitutionnel.

En comparaison, et de façon tout à fait spécifique, la Nouvelle-Calédonie prend le contrepied des situations nationales évoquées précédemment. En effet, la Nouvelle-Calédonie présente une configuration totalement inverse de ses voisins dans la mesure où l'émancipation institutionnelle a lieu au sein de la République française et en l'absence d'émancipation politique, faute d'accéder à l'indépendance.

Néanmoins, vu par le prisme interne, les démarches d'appropriation de l'organisation institutionnelle et politique peuvent être appréhendées de manière convergente. Dans le cas de la Nouvelle-Calédonie, on peut certainement emprunter à F. Hourquebie la notion de «constitutionnalisme alternatif » ${ }^{37}$ pour parler des accords de Matignon-Oudinot de 1988 et de Nouméa de 1998. L'auteur qualifie en effet les accords de sortie de crise, dont la nature est fondamentalement politique, de constitution alternative ou provisoire, non adoptée dans la forme constitutionnelle. A cet égard, l'approbation des accords de Matignon-Oudinot par référendum national et de l'accord de Nouméa par référendum local renforce la pertinence de cette appréhension. D'autant plus que, comme le souligne F. Hourquebie, le contenu de tels

\footnotetext{
${ }^{37}$ F. Hourquebie, «La construction de l'avenir : données contextuelles et cahier des charges constitutionnel », », in Transitions constitutionnelles et Constitutions transitionnelles - Quelles solutions pour une meilleure gestion des fins de conflit?, p. 47 et s.
} 
accords revêt une force inconditionnelle alors que leur contenu remet le plus souvent en cause des principes constitutionnels fondateurs. ${ }^{38}$

Tel est bien le cas de l'accord de Nouméa. Si cet accord politique a bien été constitutionnalisé pour déroger à certains principes fondateurs de la République française, on constate aujourd'hui que dans le cadre du litige relatif au corps électoral gelé pour les élections locales, c'est la lettre et l'esprit de l'accord de Nouméa qui prévaut sur la lettre de la Constitution.

D'ailleurs, B. Gueye et M. P. Tine ne disent pas autre chose lorsqu'ils expliquent que «la pratique constitutionnelle africaine considère que la constitution matérielle prévaut sur la constitution formelle » et que «les accords de paix présentent une valeur prétendument supra constitutionnelle $»^{39}$.

On le voit, malgré la différence de situation, là encore, l'évolution institutionnelle calédonienne est inscrite dans la durée par le biais d'accords politiques conclus pour une durée de dix, puis vingt années.

La société calédonienne semble aujourd'hui aspirer à une pérennisation de son statut constitutionnel et politique, que celui-ci s'inscrive dans le cadre de la République française ou en dehors.

Dans ce cadre, et à l'image des processus utilisés par ses voisins, la Nouvelle-Calédonie peut se donner le temps et rechercher le consensus pour enfin déterminer un statut pérenne la mettant à même de favoriser la construction nationale et la cohésion sociale, car beaucoup reste à faire. Si la période de vingt années d'application de l'accord de Nouméa n'a pas été mise à profit par les responsables politiques calédoniens et l'Etat pour réfléchir au "jour d'après », apparait aujourd'hui dans le débat public la nécessité de devancer l'échéance référendaire pour s'interroger sur les évolutions institutionnelles nécessaires, dont une bonne part peuvent être envisagées en faisant abstraction du résultat de la consultation d'autodétermination. A cet égard, il apparait que la stabilité du régime politique à long terme passe nécessairement par une appropriation de celui-ci par la société calédonienne. Elle ne pourra être obtenue sans un processus inclusif et participatif, qui nécessite de se donner le temps : le temps de former la population aux enjeux, le temps d'échanger avec la population et ensuite le temps de décider au sein d'une structure qui formalisera les choix formulés par la population calédonienne. C'est seulement ainsi que le «jour d'après » sera synonyme de paix civile et de cohésion sociale.

L'imminence du scrutin d'autodétermination ne saurait justifier de s'exonérer de cette phase essentielle à la réussite du processus de transition vers un statut pérenne. Là encore, le droit comparé peut être mobilisé pour imaginer des solutions. Ainsi, si la consultation des populations concernées devait aboutir à une accession de la Nouvelle-Calédonie à l'indépendance, il serait possible d'opter pour la solution d'une constitution intérimaire comme en Afrique du sud, permettant de prendre le temps de construire la Constitution de

\footnotetext{
${ }^{38}$ F. Hourquebie, op. cit.

${ }^{39}$ B. Gueye, P. M. Tine, «La légitimité et la légitimation de la transition constitutionnelle par les élections en Afrique», in Transitions constitutionnelles et Constitutions transitionnelles - Quelles solutions pour une meilleure gestion des fins de conflit?, p. 201.
} 
l'Etat nouvellement créé. Au cas contraire, un réponse négative à l'émancipation de la Nouvelle-Calédonie n'impliquerait pas nécessairement l'adoption hâtive d'un nouveau statut d'autonomie, dans la mesure où le point 5 alinéa 5 de l'accord de Nouméa prévoit que «Tant que les consultations n'auront pas abouti à la nouvelle organisation politique proposée, l'organisation politique mise en place par l'accord de 1998 restera en vigueur, à son dernier stade d'évolution, sans possibilité de retour en arrière, cette "irréversibilité " étant constitutionnellement garantie ». 